\title{
Inclusive $\pi^{0}$ and $K_{S}^{0}$ in $\gamma \gamma$ reactions at L3
}

\author{
Pablo Achard* \\ University of Geneva, DPNC, 24 quai E. Ansermet, 1205 Geneva, Switzerland. \\ * on behalf of the L3 collaboration
}

\begin{abstract}
The $\mathrm{e}^{+} \mathrm{e}^{-} \rightarrow \mathrm{e}^{+} \mathrm{e}^{-} \pi^{0}+\mathrm{X}$ and $\mathrm{e}^{+} \mathrm{e}^{-} \rightarrow \mathrm{e}^{+} \mathrm{e}^{-} \mathrm{K}_{\mathrm{S}}^{0}+\mathrm{X}$ reactions are studied at LEP using data collected with the L3 detector at $\sqrt{s}=189-202 \mathrm{GeV}$. Preliminary results for the differential cross section $d \sigma / d p_{T}$ in the transverse momentum range $0.2 \mathrm{GeV}<\mathrm{p}_{\mathrm{T}}<7.5 \mathrm{GeV}$ at central values of pseudo-rapidity, $|\eta|<0.5$ or $|\eta|<1.5$, and the differential cross section $d \sigma / d|\eta|$ for different values of $p_{T}$ are presented. For $p_{T} \leq 1 \mathrm{GeV}$, the $p_{T}$-dependence of the data are well described by an exponential fit. For $p_{T}>1 \mathrm{GeV}$ the cross sections are compared to NLO QCD calculations and to LO Monte Carlo predictions.
\end{abstract}

\section{INTRODUCTION}

Two-photon collisions are the main source of hadrons at LEP via the process $\mathrm{e}^{+} \mathrm{e}^{-} \rightarrow \mathrm{e}^{+} \mathrm{e}^{-} \gamma^{*} \gamma^{*} \rightarrow \mathrm{e}^{+} \mathrm{e}^{-}$hadrons. In the Vector Dominance Model (VDM), each photon can fluctuate into a vector meson with the same quantum numbers as the photon, thus initiating a strong interaction process with characteristics similar to hadron-hadron interactions. This process dominates in the "soft" region, where hadrons are produced with a low transverse momentum $p_{T}$. Hadrons with high $p_{T}$ are produced by the QED direct process, $\gamma^{*} \gamma^{*} \rightarrow q \bar{q}$, or by QCD processes originating from the hadronic content of the photon. Depending on wether one or two photons fluctuate into a hadronic system, the QCD processes are called single-resolved or double-resolved respectively. In two-photon interactions, QCD calculations are available for single particle inclusive production at Next-to-leading order (NLO) [1].

In this paper the inclusive $\pi^{0}$ production and the inclusive $K_{S}^{0}$ production from quasi-real photons are measured. The center-of-mass energy of the two interacting photons, $W_{\gamma \gamma}$, is greater than $5 \mathrm{GeV}$ for $\pi^{0}$ and greater than $10 \mathrm{GeV}$ for $K_{S}^{0}$. The $\pi^{0}$ is measured in the transverse momentum range $0.2 \leq p_{T} \leq 7.5 \mathrm{GeV}$ and in the pseudo-rapidity interval $-.5 \leq \eta \leq .5$, with $\eta=-\ln (\tan \theta / 2)$. The angle $\theta$ is the polar angle of the $\pi^{0}$, relative to the beam axis. The $K_{S}^{0}$ is measured in the range $0.2 \leq p_{T} \leq 4 \mathrm{GeV}$ and $-1.5 \leq \eta \leq 1.5$

The differential cross sections are compared to the Monte Carlo models PHOJET [2] and PYTHIA [3] and to analytical NLO QCD calculations [1]. 


\section{EVENT SELECTION}

The data used for this analysis were collected by the L3 detector [4] in 1998 and 1999 at beam energies from $189 \mathrm{GeV}$ to $202 \mathrm{GeV}$, with an average energy value of $194 \mathrm{GeV}$. The integrated luminosity is $414 \mathrm{pb}^{-1}$.

The $\mathrm{e}^{+} \mathrm{e}^{-} \rightarrow \mathrm{e}^{+} \mathrm{e}^{-}$hadrons processes are simulated with the PHOJET ${ }^{1}$ and PYTHIA ${ }^{2}$ event generators. For the background the annihilation processes $\mathrm{e}^{+} \mathrm{e}^{-} \rightarrow$ hadrons $(\gamma), \operatorname{ZZ}(\gamma)$, Zee $(\gamma)$, We $\nu(\gamma)$ are simulated with PYTHIA [3]; KORALZ [5] is used for $\mathrm{e}^{+} \mathrm{e}^{-} \rightarrow \tau^{+} \tau^{-}(\gamma)$ and KORALW [6] for $\mathrm{e}^{+} \mathrm{e}^{-} \rightarrow \mathrm{W}^{+} \mathrm{W}^{-}$. For the $\mathrm{e}^{+} \mathrm{e}^{-} \rightarrow \mathrm{e}^{+} \mathrm{e}^{-} \tau^{+} \tau^{-}$channel the generator DIAG36 [7] is used. The events are simulated in the L3 detector using the GEANT [8] and GEISHA [9] programs and passed through the same reconstruction program as the data.

The selection of $\mathrm{e}^{+} \mathrm{e}^{-} \rightarrow \mathrm{e}^{+} \mathrm{e}^{-}$hadrons events is based on information from the central tracking detectors and from the electromagnetic (BGO) and hadronic calorimeters. The following cuts are applied:

1. To reject annihilation events, the total energy in the calorimeters is required to be less than $40 \%$ of the center-of-mass energy.

2. To exclude radiative events of the type $\mathrm{e}^{+} \mathrm{e}^{-} \rightarrow \gamma Z \rightarrow \gamma \mathrm{Z} \overline{\mathrm{q}}$, the total energy in the electromagnetic calorimeter must be lower than $50 \mathrm{GeV}$.

3. Hadronic events are selected with at least 6 particles in the detector. These particles can be charged tracks or isolated clusters in the calorimeters.

4. Quasi-real two-photon interactions are selected by excluding events with a cluster of energy greater than $70 \mathrm{GeV}$ and polar angle greater than $33 \mathrm{mrad}$.

5. Beam-gas and beam-wall events are suppressed by the requirement that at least $500 \mathrm{MeV}$ must be deposited in the electromagnetic calorimeter.

6 . The analysis is limited to events with a visible hadronic mass $W_{\text {vis }}>5 \mathrm{GeV}$, to allow comparisons with the Monte Carlo generators which generate events with $W_{\gamma \gamma}>3 \mathrm{GeV}$. $W_{\text {vis }}$, is the effective mass of the hadronic system, calculated from the four-momentum vector of all the measured particles ${ }^{3}$.

After these cuts, the background $(\sim 1 \%)$ is due to $\mathrm{e}^{+} \mathrm{e}^{-} \rightarrow$ hadrons and $\mathrm{e}^{+} \mathrm{e}^{-} \rightarrow$ $\mathrm{e}^{+} \mathrm{e}^{-} \tau^{+} \tau^{-}$events.

Inclusive $\pi^{0}$ production is studied via the $\pi^{0}$ decay into two resolved photons. A photon is defined as an electromagnetic cluster formed by the energy deposited in at least 2 BGO crystals, with energy greater than $100 \mathrm{MeV}$ and separated by more than $200 \mathrm{mrad}$ from the closest track. The number of photons detected is required to be at least 2 but not more than 20 .

1) PHOJET version $1.05 \mathrm{c}$

2) PYTHIA version 5.718 and JETSET version 7.408

3) All particles are considered to be pions, except for isolated clusters in the electromagnetic calorimeter which are considered to be photons. 
Almost $210^{6}$ events are selected, leading to more than $810^{7}$ two photon combinations. The distribution of the mass of the reconstructed $\gamma \gamma$ system shows a narrow $\pi^{0}$ peak. A fit of this peak with a gaussian function over a Chebyshev polynomial background gives a resolution of $7.29 \pm 0.03 \mathrm{MeV}$.

Inclusive $K_{S}^{0}$ production is studied via the $K_{S}^{0}$ decay into $\pi^{+} \pi^{-}$. The distance, in the transverse plane, between the secondary vertex and the $\mathrm{e}^{+} \mathrm{e}^{-}$interaction point is required to be greater than $3 \mathrm{~mm}$. The angle between the flight direction of the $K_{S}^{0}$ candidate (taken as that of the line between the interaction point and the secondary vertex in the transverse plane) and the total transverse momentum vector of the two outgoing tracks must be less than $0.075 \mathrm{rad}$. After this cut, around $510^{5}$ events are selected. A fit of the $K_{S}^{0}$ peak gives a resolution of $10 \pm 1$ $\mathrm{MeV}$.

\section{DATA ANALYSIS}

To evaluate the number of $\pi^{0} \mathrm{~s}$ or $K_{s}^{0} \mathrm{~s}$, on each bin of $p_{T}$ and $|\eta|$, a fit is performed using a gaussian for the signal and a Chebyshev polynomial parametrisation of the background. In the $\pi^{0}$ case, to estimate the uncertainty on the background subtraction a side-band background subtraction is also performed. A three sigma region, "the signal region", is defined around the $\pi^{0}$ peak. Two "background regions" are defined on each side of the signal region with the same width. The average number of $\gamma \gamma$ combinations in the background regions is subtracted from the number of combinations in the signal region. Both methods give consistent results.

The reconstruction efficiency is evaluated using the two Monte-Carlo generators: PHOJET and PYTHIA. The kinematic limits of the cross section measurement are defined by imposing, at the generator level, $W_{\gamma \gamma} \geq 5 \mathrm{GeV}$ for $\pi^{0}$ and $W_{\gamma \gamma} \geq 10 \mathrm{GeV}$ for $K_{S}^{0}$. The virtuality of the photon is limited to $Q^{2} \leq 1 \mathrm{GeV}^{2}$ in PYTHIA, in PHOJET, a limit $Q^{2} \leq 3 \mathrm{GeV}^{2}$ for $\pi^{0}$ and $Q^{2} \leq 8 \mathrm{GeV}^{2}$ for $K_{S}^{0}$ is imposed. We have verified with PHOJET that the results do not depend on the $Q^{2}$ cutoff. The number of $\pi^{0} / K_{S}^{0}$ reconstructed is obtained with the same procedure used for the data. As the two generators reproduce equally well the experimental distributions [10], a weighted average of the two is used to correct the data.

The level-1 trigger efficiency is evaluated by comparing the response of two independent triggers: the energy trigger [11] and the track trigger [12]. The efficiency is 90-95\%, varying with the data taking conditions during the year. The efficiencies of higher level triggers, calculated using prescaled events, vary from $85 \%$ at low $p_{T}$ to $100 \%$ at high $p_{T}$.

The main uncertainty on the measured cross sections comes from the choice of Monte Carlo generator used to calculate the reconstruction efficiency and from the background estimation. Half of the difference between the two generators and between the two estimations of the background are taken as systematic errors. The uncertainties due to cut variations are negligible. 
Statistical and systematic errors are added in quadrature in the following. All the results are preliminary.

\section{RESULTS}

The differential cross sections $d \sigma / d p_{T}$ are shown in fig.1. Exponential fits $e^{-p_{T} /<p_{T}>}$ for $0.2 \mathrm{GeV}<\mathrm{p}_{\mathrm{T}}<1.0 \mathrm{GeV}$ reproduce well the data with $\left\langle p_{T}>=\right.$ $233 \pm 1 \mathrm{MeV}$ for $\pi^{0}$ and $\left\langle p_{T}\right\rangle=357 \pm 9 \mathrm{MeV}$ for $K_{S}^{0}$. This behaviour is characteristic of hadrons produced by soft interactions and the fitted values of $\left\langle p_{T}\right\rangle$ are similar to the one obtained in hadron-hadron or photon-hadron collisions [13].

Due to the existence of the direct process and of hard QCD interactions, $\gamma^{*} \gamma^{*}$ collisions start to exhibit a higher cross section at $p_{T}$ values greater than $\sim 1.5 \mathrm{GeV}$. In fig. 2 and 3 the $d \sigma / d p_{T}$ and $d \sigma / d|\eta|$ differential cross sections are compared to analytical NLO QCD predictions [1]. For this calculation, the flux of quasi-real photons is obtained using the Equivalent Photon Approximation, taking into account both transverse and longitudinal virtual photons. The interacting particles can be photons or partons from the $\gamma \rightarrow q \bar{q}$ quantum fluctuation, which evolves, via the Altarelli-Parisi equation, into quarks and gluons. The Gordon and Storrow [14] parton density functions are used. All elementary $2 \rightarrow 2$ and $2 \rightarrow 3$ processes are considered. The renormalization scale, the factorisation scale and the fragmentation scale are taken to be equal: $\mu=M=M_{F}=\xi p_{T}$. The uncertainty in the NLO calculation is estimated by varying the value of $\xi$ from 0.5 to 2.0 .

The measured cross sections are one order of magnitude above the direct process predictions. In the $\pi^{0}$ case, for $p_{T}>2 \mathrm{GeV}$, the data are also higher than the predictions of NLO QCD calculations.
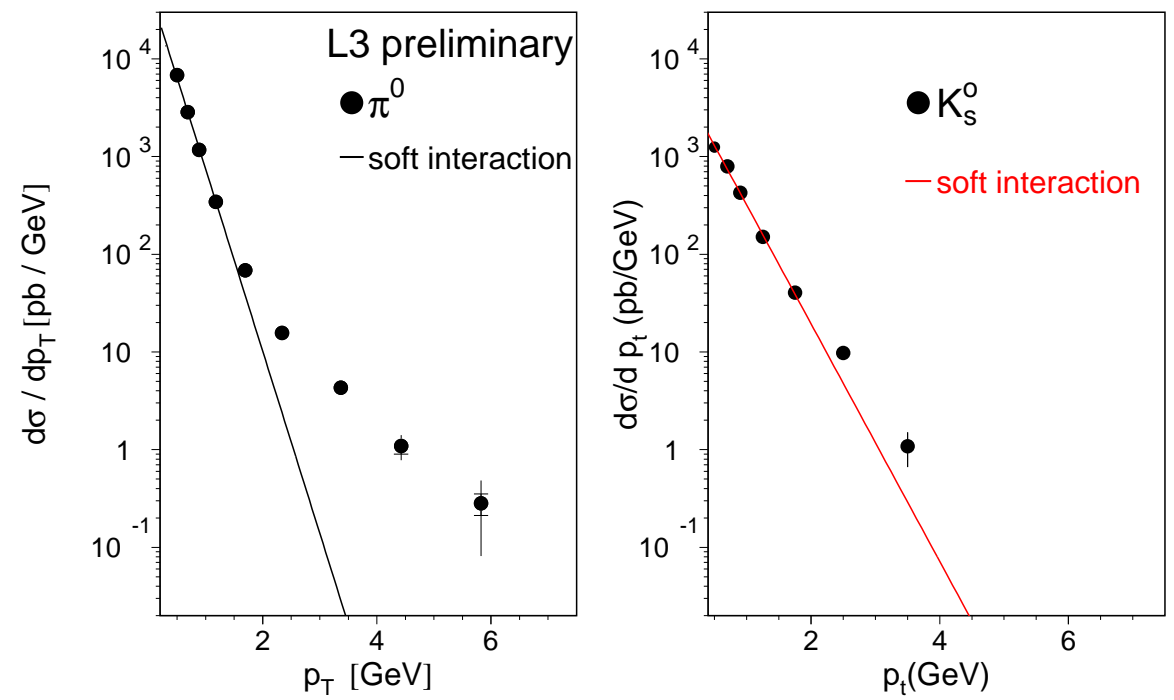

FIGURE 1. The differential cross sections $d \sigma / d p_{T}$ as a function of $p_{T}$ for $\pi^{0}$ and $K_{S}^{0}$. The low $p_{T}$ spectrum is well reproduced by an exponential fit, characteristic of soft interactions. 

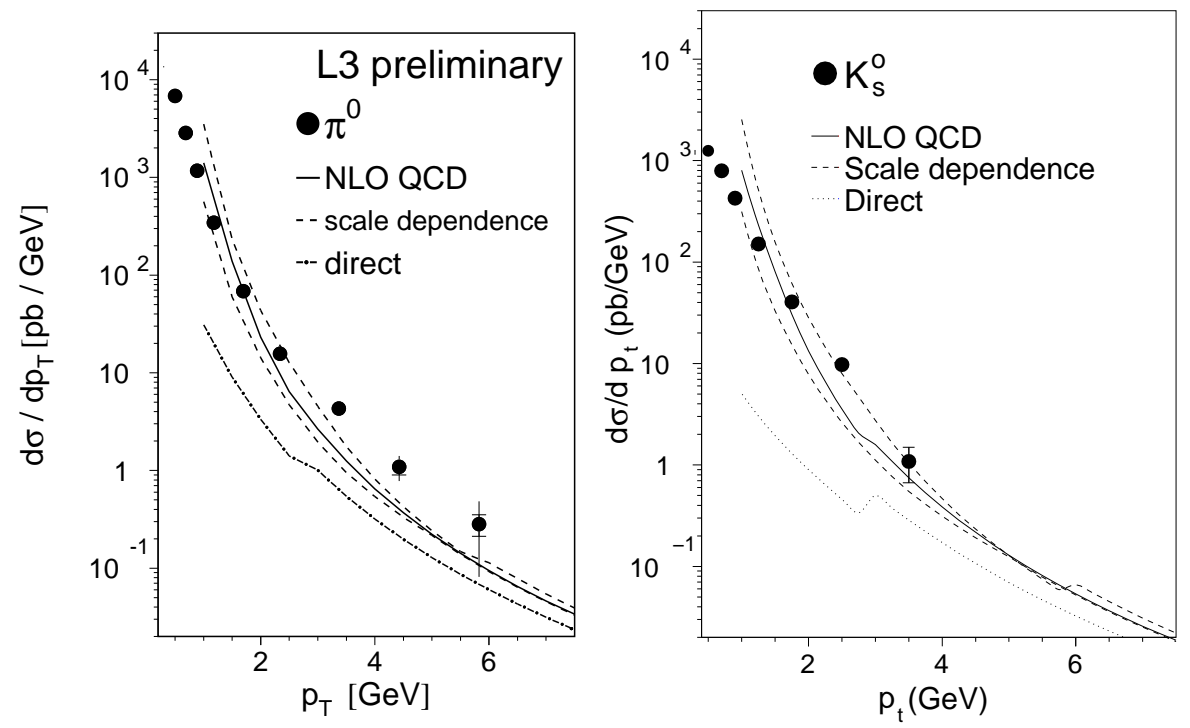

FIGURE 2. Comparison of the $d \sigma / d p_{T}$ distributions with NLO-QCD calculations. The central values (full lines) are the calculations with $\xi=1$ (see text), the dashed lines refer to $\xi=0.5$ (upper lines) and to $\xi=2$ (lower lines). The contribution of the direct process alone are indicated with dash-dotted lines.

The cross sections are also compared to Monte Carlo predictions in fig.4. The high $p_{T}$ region is better reproduced by PYTHIA than by PHOJET in the $\pi^{0}$ case. For the $K_{S}^{0}$ both Monte-Carlos reproduce quite well the data.

The differential cross sections, calculated for $W_{\gamma \gamma}>10 \mathrm{GeV}$, are compared to OPAL results [15] in fig.5. OPAL measured the inclusive production of charged hadrons (mainly $\pi^{ \pm}$) and of $K_{S}^{0}$ in the range $|\eta|<1.5$. The experiments agree within the experimental errors.
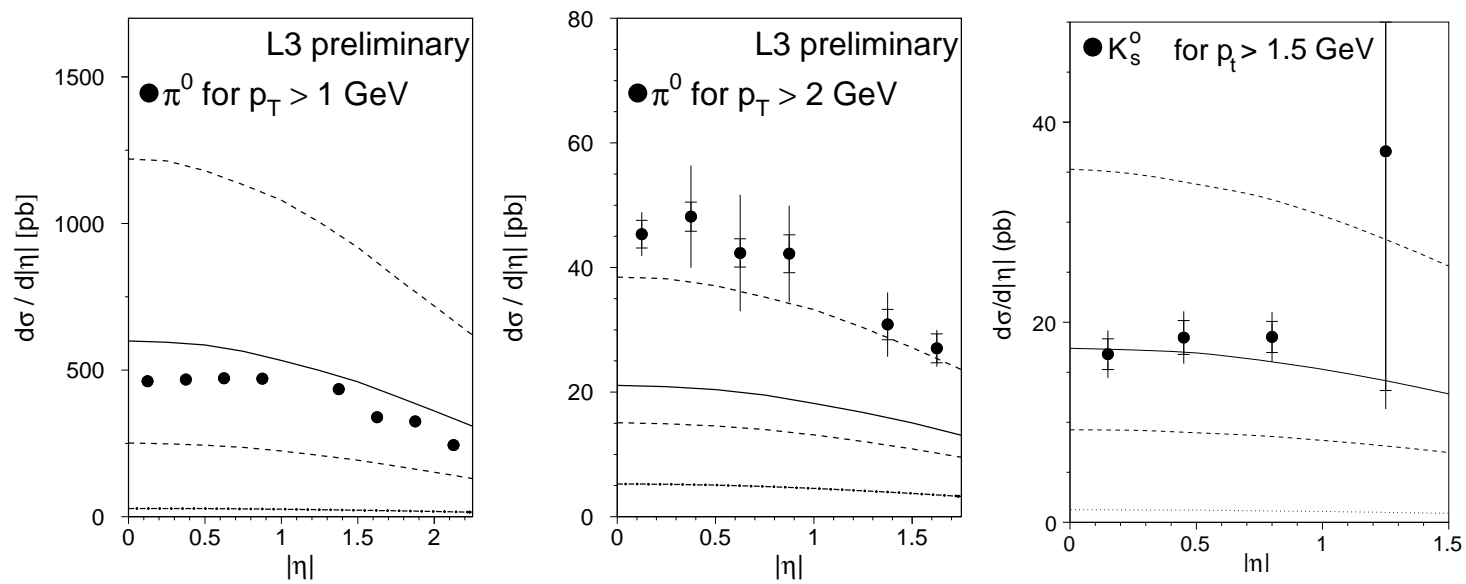

FIGURE 3. Comparison of the $d \sigma / d|\eta|$ distributions with NLO-QCD calculations. The lines are as in fig.2. 

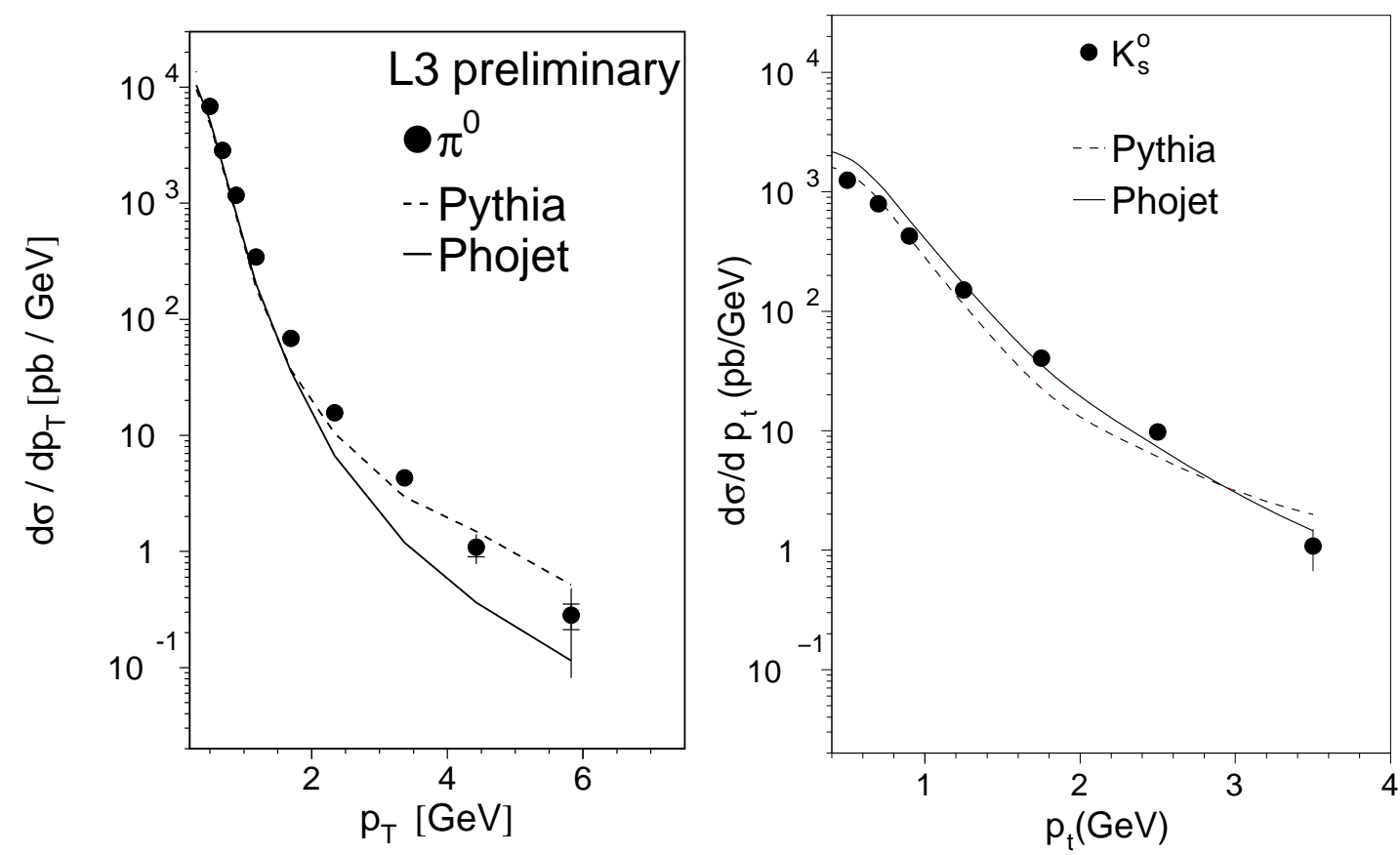

FIGURE 4. Comparison of the $d \sigma / d p_{T}$ distributions with Monte Carlo predictions.
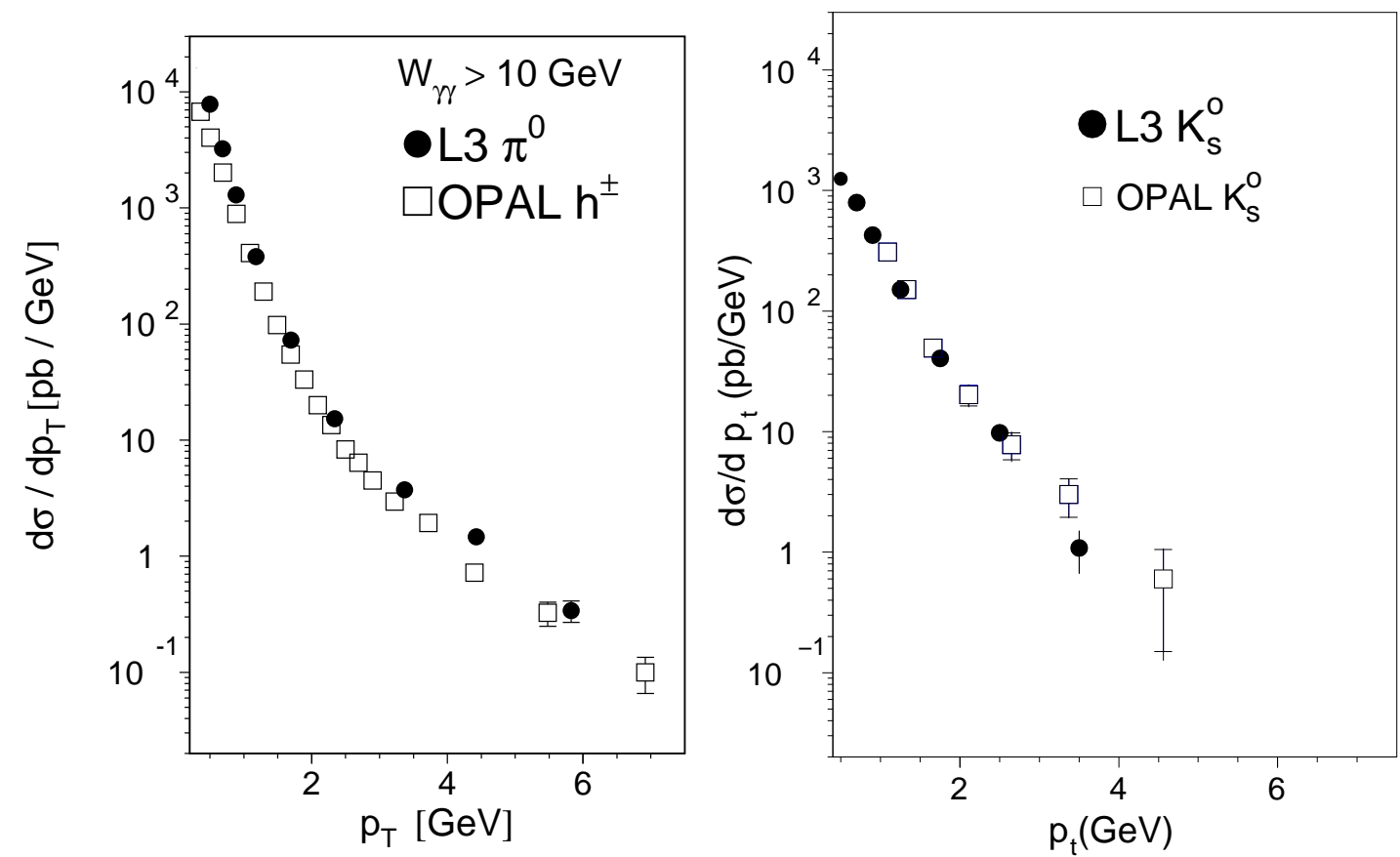

FIGURE 5. The L3 and OPAL $d \sigma / d p_{T}$ distributions compared. The OPAL data are scaled to the same center-of-mass energy and, in the $\pi^{0}$ vs. charged hadron case, are scaled also to the same pseudorapidity range and fragmentation function. 


\section{CONCLUSIONS}

This analysis is the first study of inclusive $\pi^{0}$ production in two photon collisions at LEP. At low $p_{T}$, the differential cross sections $d \sigma / d p_{T}$ of $\pi^{0}$ and $K_{S}^{0}$ production follow an exponential law similar to that previous observed in hadron-hadron and hadron-photon collisions. At higher $p_{T}$, the contributions of $\gamma \gamma \rightarrow q \bar{q}$ and hard QCD processes are clearly visible. The agreement with previous measurement is very good, but there is only a qualitative agreement with the predictions of LO Monte Carlo and NLO QCD calculations.

\section{ACKNOWLEDGMENTS}

I would like to thank B.A. Kniehl for providing us the predictions of NLO QCD calculations and L. Gordon for very useful discussions.

Thanks a lot also to A. Finch and all the Photon 2000 team for the kindness and efficiency of the organization.

Many thanks, of course, to S. Braccini for providing me the $K_{S}^{0}$ results.

\section{REFERENCES}

1. Binnewies, J., Kniehl, B.A., and Kramer, G. Phys. Rev. D 53, 6110 (1996).

2. Engel, R. , Z. Phys. C 66, 203 (1995);

Engel, R., and Ranft, J., Phys. Rev. D 54, 4246 (1996);

And Engel, R., private communication.

3. Sjöstrand, T., Comput. Phys. Commun. 82, 74 (1994).

4. L3 Coll., Adeva, B., et al., N.I.M. A 289, 35 (1990);

L3 Coll., Acciarri, M., et al., N.I.M. A 351, 300 (1994).

5. Jadach, S., Ward, B. F. L., and Was, Z., Comput. Phys. Commun. 79, 503 (1994).

6. Skrzypek, M., Jadach, S., Placzek, W. and Was, Z., Comput. Phys. Commun. 94, 216 (1996).

7. Berends, F.A., Daverfeldt, P.H., and Kleiss, R., Nucl. Phys. B 253, 441 (1985).

8. Brun, R., et al., GEANT 3.15 preprint CERN DD/EE/84-1 (Revised 1987).

9. Fesefeldt, H., RWTH Aachen report PITHA 85/2 (1985).

10. Kienzle, M.N., these proceedings.

11. Bizzarri, R., et al. , N.I.M. A 283, 799 (1989).

12. Béné, P., et al., N.I.M. A 306, 150 (1991);

Haas, D., et al., N.I.M. A 420, 101 (1999).

13. Perl, M.L., High Energy Hadron Physics, edited by J. Wiley, 1974.

14. Gordon, L.E., and Storrow, J.K., Nucl. Phys. B 489, 405 (1997).

15. OPAL Coll., Abbiendi, G., et al., Eur. Phys. J. C 6, 253 (1999). 